\title{
Qubit thermometry for micromechanical resonators
}

\author{
Matteo Brunelli* \\ Dipartimento di Fisica, Università degli Studi di Milano, I-20133 Milano, Italy \\ Stefano Olivares ${ }^{\dagger}$ \\ Dipartimento di Fisica, Università degli Studi di Trieste, I-34151 Trieste, Italy and \\ CNISM UdR Milano Statale, I-20133 Milano, Italy \\ Matteo G. A. Paris \\ Dipartimento di Fisica, Università degli Studi di Milano, I-20133 Milano, Italy and \\ CNISM UdR Milano Statale, I-20133 Milano, Italy \\ (Received 18 March 2011; published 8 September 2011)
}

\begin{abstract}
We address estimation of temperature for a micromechanical oscillator lying arbitrarily close to its quantum ground state. Motivated by recent experiments, we assume that the oscillator is coupled to a probe qubit via Jaynes-Cummings interaction and that the estimation of its effective temperature is achieved via quantum-limited measurements on the qubit. We first consider the ideal unitary evolution in a noiseless environment and then take into account the noise due to nondissipative decoherence. We exploit local quantum estimation theory to assess and optimize the precision of estimation procedures based on the measurement of qubit population and to compare their performances with the ultimate limit posed by quantum mechanics. In particular, we evaluate the Fisher information (FI) for population measurement, maximize its value over the possible qubit preparations and interaction times, and compare its behavior with that of the quantum Fisher information (QFI). We found that the FI for population measurement is equal to the QFI, i.e., population measurement is optimal, for a suitable initial preparation of the qubit and a predictable interaction time. The same configuration also corresponds to the maximum of the QFI itself. Our results indicate that the achievement of the ultimate bound to precision allowed by quantum mechanics is in the capabilities of the current technology.
\end{abstract}

DOI: 10.1103/PhysRevA.84.032105

PACS number(s): 03.65.Ta, 42.50.-p

\section{INTRODUCTION}

The edge between classical and quantum description of a phenomenon is related to the interactions occurring between the system under investigation and its environment. As a consequence, if we could, in ideal conditions, avoid irreversible interactions among them, we should observe the emergence of quantum behavior even in macroscopic systems. As a matter of fact, the technological developments in recent years have made it possible to start inquiring into the quantum limit even in mesoscopic mechanical systems, and experiments have been designed that realize a solid-state analog of cavity quantum electrodynamics. Many of these experiments focus on detecting the quantization of vibrational modes in a mechanical oscillator [1-11]. Experimental conditions such that a mechanical object may behave in a quantum fashion are achieved in the low-temperature regime. For example, for a single vibrational mode of energy $\hbar \omega$ to show quantum features, such as the quantization of lattice vibrations, temperatures $T \ll \frac{\hbar \omega}{k_{B}}$ are required, which for a microsized object oscillating in the microwave band correspond to a few $\mathrm{mK}$.

In this framework it has become increasingly relevant to have a precise determination of the temperature. However, for a quantum system in equilibrium with a thermal bath, there is no linear operator that acts as an observable for temperature.

\footnotetext{
*matteo.brunelli@studenti.unimi.it

${ }^{\dagger}$ stefano.olivares@ts.infn.it

${ }^{\ddagger}$ matteo.paris@ fisica.unimi.it
}

Temperature, thought to be a macroscopic manifestation of random energy exchanges between particles, still retains its meaning, but we have lost any operational definition. This kind of impediment often occurs in physics, especially in quantum mechanics, whenever one is interested in quantities that are not directly accessible, i.e., they do not correspond to observable quantities. This may either be due to experimental impossibilities or be a matter of principle, as it happens for nonlinear functions of the density operator. In both cases, it turns out that the only way to gain some knowledge about the quantity of interest is to measure one or more proper observables somehow related to the parameter we are interested in and, upon suitably processing the outcomes, to come back and infer its value. Hence, any conceivable strategy aimed at evaluating the quantity of interest ultimately reduces to a parameter-estimation problem. Relevant examples of this situation are given by estimation of the quantum phase of a harmonic oscillator [12-15], the amount of entanglement of a bipartite quantum state [16-18], and the coupling constants of different kinds of interactions [19-29]. Here we focus on the estimation of temperature [30] and, motivated by recent experimental achievements [11], we specifically refer to schemes where a micromechanical resonator is coupled to a superconducting qubit, and then a measurement of the excited state population is performed on the qubit itself. From the statistics of the population measurement it is then possible to obtain information about the oscillator state, e.g., infer how close it is to the ground state and, in turn, its temperature.

In this context an optimization problem naturally arises, aimed at finding the most efficient inference procedure leading 
to minimum fluctuations in the temperature estimate. In this paper we address this problem in the framework of local quantum estimation theory (QET) [31-36]. We solve the dynamics of the qubit-resonator coupled system, and in order to match realistic scenarios, we also take into account an effective model for nondissipative decoherence. Then, we evaluate the Fisher information (FI) for the estimation of temperature via population measurement (hereafter referred to as the FI of the population measurement) and find both the optimal initial qubit preparation and the smallest temperature value that can be discriminated. Moreover, we evaluate the quantum Fisher information (QFI) in terms of the symmetric logarithmic derivative in order to calculate the ultimate bound to the precision allowed by quantum mechanics. This enable us to show that population measurement is indeed optimal for a suitable choice of the initial preparation of the qubit and, more generally, to provide quantum benchmarks for temperature estimation.

It is worth noting at this point that we are not discussing here temperature fluctuations in a thermodynamical setting. Although temperature itself may not fluctuate, as suggested by quantum thermodynamical approaches [37], we expect that fluctuations always appear in the temperature estimates coming from indirect measurements [38,39]. Quantum estimation theory provides the tools to evaluate lower bounds to the amount of fluctuations for a given measurement, as well as the ultimate bounds imposed by quantum mechanics.

This paper is structured as follows. In Sec. II we describe the interaction model: first, we briefly review the unitary JaynesCummings dynamics for the coupled system and describe the measurements performed on the qubit, and then we take into account the decoherence effects. In Sec. III we show how QET techniques apply to our system, providing explicit formulas for both the FI of the population measurement and the QFI. The results are finally shown in detail in Sec. IV both for the unitary and the noisy dynamics. Sec. V closes the paper with some concluding remarks.

\section{THE PHYSICAL MODEL}

As the temperature decreases, a mechanical oscillator starts to exhibit its quantum nature, which mainly manifests itself in quantization of the vibrational modes. Hence, for our purposes the resonator can be regarded as a collection of phonons in a thermal equilibrium state. We assume that the resonator is built to display an isolated mechanical mode at a given frequency, so that it can be modeled, rather than a phonon bath with some spectral distribution, as a single-mode phonon field in thermal equilibrium.

\section{A. Unitary dynamics}

Let $\mathcal{H}_{R}$ be the infinite dimensional Hilbert space associated with the single-mode phonon field. Upon introducing the creation and annihilation operators $\left[a, a^{\dagger}\right]=1$ one has the number operator $N=a^{\dagger} a$ and its eigenstates $\{|n\rangle\}_{n=0}^{\infty}$. The field Hamiltonian reads

$$
H_{F}=\hbar \Omega a^{\dagger} a
$$

where $\Omega$ denotes the frequency of the vibrational mode. We assume the resonator to be in a thermal equilibrium state, i.e., described by the density operator

$$
\varrho_{F}=\frac{\exp \left(-\beta H_{F}\right)}{\operatorname{Tr}\left[\exp \left(-\beta H_{F}\right)\right]}=\sum_{n=0}^{\infty} p_{n}(\Omega, \beta)|n\rangle\langle n|,
$$

where $\beta=\left(k_{B} T\right)^{-1}$ and

$$
p_{n}(\Omega, \beta)=e^{-\beta \hbar \Omega n}\left(1-e^{-\beta \hbar \Omega}\right) .
$$

The resonator is coupled to a superconducting qubit whose initial preparation is under control, and after a given interaction time, the excited state population is detected. The qubit is treated as a normalized vector in a two-dimensional complex Hilbert space $\mathcal{H}_{Q}$, with $\{|e\rangle,|g\rangle\}$ providing an orthonormal basis. The qubit is initially prepared in a pure state,

$$
|\psi\rangle=\cos \frac{\vartheta}{2}|e\rangle+e^{i \varphi} \sin \frac{\vartheta}{2}|g\rangle,
$$

with $\varphi \in[0,2 \pi)$ and $\vartheta \in[0, \pi]$. Hence the qubit density operator reduces to the projector $\varrho_{Q}=|\psi\rangle\langle\psi|$. Being a two-level system, by appropriately choosing the zero-energy level and denoting by $\omega$ its transition frequency, the qubit Hamiltonian can be written as

$$
H_{Q}=\frac{\hbar \omega}{2} \sigma_{z}
$$

The qubit-resonator interaction is the interaction between a single-mode bosonic field and a two-level system. In the rotating-wave approximations and for the near-resonant case, i.e., for small values of the detuning $\delta=\omega-\Omega$, we have the Jaynes-Cummings (JC) model with Hamiltonian

$$
\begin{aligned}
\tilde{H}_{\mathrm{JC}} & =H_{Q}+H_{F}+H_{\mathrm{int}} \\
& =\frac{\hbar \omega}{2} \sigma_{z}+\hbar \Omega a^{\dagger} a+\hbar \lambda\left(\sigma_{+} a+\sigma_{-} a^{\dagger}\right) .
\end{aligned}
$$

The unperturbed Hamiltonian $\tilde{H}_{\mathrm{JC}}^{(0)}=H_{Q}+H_{F}$ satisfies the eigenvalues equations

$$
\tilde{H}_{\mathrm{JC}}^{(0)}|k, n\rangle=\hbar\left[n \Omega+\frac{1}{2} \omega(-1)^{k}\right]|k, n\rangle,
$$

with $k=e, g$ and with the correspondences $0 \leftrightarrow e, 1 \leftrightarrow g$. In Eq. (4) $\lambda \in \mathbb{R}$ represents the coupling strength, $\sigma_{+} a$ and $\sigma_{-} a^{\dagger}$ stand, respectively, for the operators $\sigma_{+} \otimes a$ and $\sigma_{-} \otimes a^{\dagger}$ acting on the tensor product space, where $\sigma_{ \pm}$are the qubit ladder operators. Upon choosing a suitable rotating frame one can rewrite the Hamiltonian in the interaction picture $H_{\mathrm{JC}}$ :

$$
H_{\mathrm{JC}}=\frac{\hbar \delta \sigma_{z}}{2}+\hbar \lambda\left(\sigma_{+} a+\sigma_{-} a^{\dagger}\right)
$$

The interaction only couples, for a given $n$, the states $|e, n\rangle$ and $|g, n+1\rangle$, and thus it is possible to study the interaction inside the two-dimensional manifold spanned by these states, leading to a representation, the so-called dressed-states basis, where $H_{\mathrm{JC}}$ is diagonal. We further assume the absence of any initial correlations between the qubit and the oscillator, thus choosing at time $t=0$ the following factorized density operator:

$$
\varrho(0)=\varrho_{Q} \otimes \varrho_{F},
$$

whose dynamical evolution with respect to the JC Hamiltonian is given by

$$
\varrho(t)=U(t) \varrho(0) U^{\dagger}(t),
$$

with $U(t)=\exp \left(-\frac{i}{\hbar} H_{\mathrm{JC}} t\right)$. 
Time evolution entangles the qubit and the resonator [40], and the probabilities for the qubit to be found in the ground or excited state are obtained via the Born rule as

$$
p(j \mid \beta)=\operatorname{Tr}_{Q F}\left[\varrho(t)|j\rangle\langle j| \otimes \mathbb{I}_{F}\right] \quad j=e, g,
$$

where $p(j \mid \beta)$ denotes the conditional probability of obtaining the value $j$ when the value of the temperature parameter is $\beta$. Upon introducing the quantum operation

$$
\varrho_{Q} \stackrel{\mathcal{E}}{\longmapsto} \varrho_{P} \equiv \operatorname{Tr}_{F}\left[U(t) \varrho_{Q} \otimes \varrho_{F} U^{\dagger}(t)\right],
$$

where $\mathcal{E}: \mathcal{L}\left(\mathcal{H}_{Q}\right) \rightarrow \mathcal{L}\left(\mathcal{H}_{Q}\right)$, Eq. (6) can be equally rewritten at the level of the qubit subsystem alone, namely,

$$
p(j \mid \beta)=\operatorname{Tr}_{Q}\left[\varrho_{P}|j\rangle\langle j|\right] .
$$

In the following we will refer to $\varrho_{P}$ as the probe state: It describes the qubit subsystem at time $t$, obtained as the partial trace over the phonon field of the overall evolved state of the coupled system. Since it is a density operator on $\mathcal{H}_{Q}$, it can be arranged in a $2 \times 2$ density matrix. We have

$$
\varrho_{P}=\sum_{n=0}^{\infty} p_{n}(\Omega, \beta)\left(\begin{array}{ll}
\varrho_{e e} & \varrho_{e g} \\
\varrho_{g e} & \varrho_{g g}
\end{array}\right),
$$

where

$$
\begin{gathered}
\varrho_{e e}=\cos ^{2} \frac{\vartheta}{2}\left[\cos ^{2} \theta_{n} t+4 \frac{\delta^{2}}{\theta_{n}^{2}} \sin ^{2} \theta_{n} t\right] \\
+\sin ^{2} \frac{\vartheta}{2} \frac{\lambda^{2} n}{\theta_{n-1}^{2}} \sin ^{2} \theta_{n-1} t, \\
\varrho_{e g}=\frac{1}{2} e^{-i \varphi} \sin \vartheta\left[\cos \theta_{n-1} t+i \frac{2 \delta}{\theta_{n-1}} \sin \theta_{n-1} t\right] \\
\times\left[\cos \theta_{n} t-i \frac{2 \delta}{\theta_{n}} \sin \theta_{n} t\right], \\
\varrho_{g e}=\varrho_{e g}^{*}, \quad \varrho_{g g}=1-\varrho_{e e},
\end{gathered}
$$

with

$$
\theta_{n} \equiv \theta_{n}(\delta, \lambda)=\frac{1}{2} \sqrt{\delta^{2}+4 \lambda^{2}(n+1)} .
$$

\section{B. Effects of decoherence}

A purely Hamiltonian dynamics does not describe realistic conditions. In real-life scenarios quantum coherence is hard to achieve in mechanical objects and can be maintained for relatively small times $\left(\approx 10^{-9}\right.$ s). Complete Rabi oscillations between the phonon and the qubit excitation involve only the first Rabi half periods; then a damping of the probabilities $p(j \mid \beta)$ to $\frac{1}{2}$ is observed: the most striking signature of decoherence. Hence we include in our model the treatment of nondissipative decoherence occurring between the qubit and the resonator. Following Ref. [41], we consider an effective model provided by adding a power-law term in the thermal distribution, which leads to probe state matrix elements given by

$$
\tilde{\varrho}_{i j}=\sum_{n=0}^{\infty} p_{n}(\Omega, \beta)\left[e^{-\gamma_{n} t} \varrho_{i j}+\frac{1}{2}\left(1-e^{-\gamma_{n} t}\right)\right],
$$

with $\varrho_{i j}$ being the matrix elements of Eq. (9), as evaluated for the unitary case, $i, j \in\{e, g\}$, and

$$
\gamma_{n}=b(1+n)^{a} .
$$

More explicitly,

$$
\begin{gathered}
\tilde{\varrho}_{e e}=\frac{1}{2}\left[1+\sum_{n=0}^{\infty} p_{n}(\Omega, \beta) e^{-\beta e^{-\gamma_{n} t}}\left(\varrho_{e e}-\varrho_{g g}\right)\right], \\
\tilde{\varrho}_{e g}=\frac{1}{2} \sum_{n=0}^{\infty} p_{n}(\Omega, \beta) e^{-\gamma_{n} t} \varrho_{e g}, \\
\tilde{\varrho}_{g e}=\tilde{\varrho}_{e g}^{*}, \quad \tilde{\varrho}_{g g}=1-\tilde{\varrho}_{e e} .
\end{gathered}
$$

One can see that the dynamical evolution now drives the qubit toward the maximally mixed state, described by the density operator $\frac{\mathbb{I}}{2}$.

\section{QUANTUM THERMOMETRY}

In this section we apply the tools of (local) QET to the coupled qubit-oscillator system. An estimation problem always consists of two steps: at first one has to choose a measurement, and then, after collecting a sample of outcomes, one should find an estimator, i.e., a function to process data and to infer the value of the quantity of interest. In our case, temperature, expressed as $\beta$, is the unknown parameter that has to be estimated from the sample of outcomes coming from measurements performed on the qubit. The results, a string of zeros and ones for the case of population measurement, are distributed according to the probabilities $p(j \mid \beta) \equiv \varrho_{j j}$ of Eqs. (8) and (9) [or Eq. (10) in the presence of decoherence]. The Cramér-Rao inequality establishes that the variance $\operatorname{Var}(\beta)$ of any unbiased estimator is lower bounded by

$$
\operatorname{Var}(\beta) \geqslant \frac{1}{M F(\beta)},
$$

where $M$ is the cardinality of the sample, i.e., the number of measurements, and $F(\beta)$ is the so-called Fisher information:

$$
\begin{aligned}
F(\beta) & =\sum_{j=e, g} p(j \mid \beta)\left[\partial_{\beta} \ln p(j \mid \beta)\right]^{2} \\
& =\frac{\left[\partial_{\beta} p(e \mid \beta)\right]^{2}}{p(e \mid \beta)}+\frac{\left[\partial_{\beta} p(g \mid \beta)\right]^{2}}{p(g \mid \beta)} .
\end{aligned}
$$

Efficient estimators are those saturating the Cramér-Rao inequality, and their existence depends on the statistical model. However, independently of the statistical model, we have that for sufficiently large samples, i.e., in the asymptotic regime $M \gg 1$, maximum likelihood and Bayesian estimators are always efficient.

Quantum mechanically, the probability of obtaining the outcome $j \in\{e, g\}$ from a measurement is given according to the Born rule by $p(j \mid \beta)=\operatorname{Tr}\left[\varrho_{P} \Pi_{j}\right]$, where the probe state $\varrho_{P} \equiv \varrho_{P}(\beta)$ parametrized by the unknown quantity $\beta$ is referred to as the quantum statistical model, and the collection of operators $\left\{\Pi_{j}\right\}, \Pi_{j} \geqslant 0, \sum_{j} \Pi_{j}=\mathbb{I}$ is the probability operator-valued measure describing the measurement taking place on the qubit. In our case the qubit excited state population is probed, and the measurement reduces to a projective one, 
$|e\rangle\langle e|$ and $|g\rangle\langle g|=\mathbb{I}-| e\rangle\langle e|$, i.e., we are measuring the Pauli operator $\sigma_{z}=|e\rangle\langle e|-| g\rangle\langle g|$.

Once the observable is fixed, we optimize the estimation procedure by maximizing the FI over the qubit state parameters, $\vartheta$ and $\varphi$, as well as over the parameters driving the interaction, i.e., the detuning $\delta$ and the interaction time $t$. In other words, by employing the optimal qubit preparation and tuning the interaction parameters one may find a working regime achieving the maximum precision for that kind of measurement.

On the other hand, one may also maximize the FI over all possible quantum measurements. Upon defining the symmetric logarithmic derivative (SLD) $L_{\beta}$ as the self-adjoint operator satisfying the equation

$$
\frac{L_{\beta} \varrho_{P}+\varrho_{P} L_{\beta}}{2}=\partial_{\beta} \varrho_{P},
$$

it is possible to show that the Fisher information $F(\beta)$ of any quantum measurement is upper bounded by the following quantity:

$$
F(\beta) \leqslant G(\beta) \equiv \operatorname{Tr}\left[\varrho_{P} L_{\beta}^{2}\right],
$$

which is called quantum Fisher information. QFI does not depend on the measurement carried on the qubit, indeed being obtained by maximizing over the possible measurement. It is rather an attribute of the family of states $\varrho_{P}(\beta)$ parametrized by the temperature. Looking back to the Cramér-Rao inequality, Eq. (11), one sees that QFI allows one to write its natural quantum version

$$
\operatorname{Var}(\beta) \geqslant \frac{1}{M G(\beta)} .
$$

The above equation represents the quantum Cramér-Rao bound (QCR), i.e., the ultimate bound to the precision allowed by quantum mechanics for a given statistical model $\varrho_{P}(\beta)$. An optimal measurement, i.e., a measurement whose FI $F(\beta)=G(\beta)$ equals the QFI for the parameter $\beta$, is given by the observable corresponding to the spectral measure of the SLD $L_{\beta}$. On the other hand, other kinds of measurements may achieve optimality for the whole range of values of $\beta$ or for a subset of values. Indeed, we will see in the following that population measurement is optimal for a suitable choice of the initial qubit preparation. We recall that for the estimation of a single parameter, as in our case, the QCR may be always attained, and an estimator saturating inequality (15) is called efficient. As in the classical case, the existence of an efficient estimator depends on the statistical model. However, independently of the statistical model, for sufficiently large samples, i.e., in the asymptotic regime $M \gg 1$, maximum likelihood and Bayesian estimators are always efficient.

Upon diagonalizing the probe state one achieves the decomposition $\varrho_{P}=\varrho_{+}\left|\psi_{+}\right\rangle\left\langle\psi_{+}\left|+\varrho_{-}\right| \psi_{-}\right\rangle\left\langle\psi_{-}\right|$and is able to solve the equation for SLD,

$$
\begin{aligned}
L_{\beta}= & \frac{\left\langle\psi_{+}\left|\partial_{\beta} \varrho_{P}\right| \psi_{+}\right\rangle}{\varrho_{+}}\left|\psi_{+}\right\rangle\left\langle\psi_{+}\right| \\
& +\frac{\left\langle\psi_{-}\left|\partial_{\beta} \varrho_{P}\right| \psi_{-}\right\rangle}{\varrho_{-}}\left|\psi_{-}\right\rangle\left\langle\psi_{-}\right| \\
& +\frac{2}{\varrho_{+}+\varrho_{-}}\left[\left\langle\psi_{+}\left|\partial_{\beta} \varrho_{P}\right| \psi_{-}\right\rangle\left|\psi_{+}\right\rangle\left\langle\psi_{-}\right|\right. \\
& \left.+\left\langle\psi_{-}\left|\partial_{\beta} \varrho_{P}\right| \psi_{+}\right\rangle\left|\psi_{-}\right\rangle\left\langle\psi_{+}\right|\right],
\end{aligned}
$$

finally obtaining an explicit formula for the QFI,

$$
\begin{aligned}
G(\beta)= & \frac{\left(\partial_{\beta} \varrho_{+}\right)^{2}}{\varrho_{+}}+\frac{\left(\partial_{\beta} \varrho_{-}\right)^{2}}{\varrho_{-}} \\
& +2 \kappa\left[\left|\left\langle\psi_{-} \mid \partial_{\beta} \psi_{+}\right\rangle\right|^{2}+\left|\left\langle\psi_{+} \mid \partial_{\beta} \psi_{-}\right\rangle\right|^{2}\right],
\end{aligned}
$$

where

$$
\left|\partial_{\beta} \psi_{ \pm}\right\rangle=\partial_{\beta}\left\langle e \mid \psi_{ \pm}\right\rangle|e\rangle+\partial_{\beta}\left\langle g \mid \psi_{ \pm}\right\rangle|g\rangle
$$

and

$$
\kappa=\frac{\left(\varrho_{+}-\varrho_{-}\right)^{2}}{\varrho_{+}+\varrho_{-}}=\left(1-2 \varrho_{+}\right)^{2} .
$$

Equation (17) contains a first term that resembles the FI and a second one, truly quantum in nature, that leads to the QCR and vanishes whenever $\left|\psi_{ \pm}\right\rangle$does not depend on $\beta$.

\section{DYNAMICS OF THE FISHER INFORMATION AND OPTIMAL WORKING REGIMES}

In this section we report results for the qubit-resonator coupled system with physical parameters chosen in a range matching the experimental setup of Ref. [11]. More specifically, we present a systematic study of the FI for population measurement as a function of the state and interaction parameters, carrying out numerical maximization and finding the optimal working regimes. We also evaluate the QFI of the family of states $\varrho_{P}(\beta)$ and find the ultimate bound to precision, i.e., a benchmark in order to assess the performances of qubit thermometry via population measurement.

Hereafter we work with dimensionless quantities by rescaling times and frequencies in units of the coupling $\lambda$. We thus substitute time, detuning, and decoherence parameters by their rescaled counterparts

$$
t \longmapsto \tau \equiv \lambda t, \quad \delta \longmapsto \gamma \equiv \delta / \lambda, \quad b \longmapsto \tilde{b} \equiv b / \lambda .
$$

Effective detuning $\gamma$ will range in $|\gamma| \in[0,1.5]$. Also, a dimensionless effective temperature $\tilde{\beta}$ is defined, provided by the substitution

$$
\beta \longmapsto \tilde{\beta} \equiv \beta \hbar \Omega .
$$

For convenience, we continue to term $\tilde{\beta}$ and $\tilde{b}$, respectively, as $\beta$ and $b$.

\section{A. Resonant Hamiltonian regime}

Upon using the expression of the diagonal matrix elements in Eqs. (9) we have evaluated the FI of Eq. (12). We start the discussion by considering the resonant case, i.e., zero detuning, and analyze the effect of detuning afterward in this section. For convenience we adopt the notation $F(\beta)$ for the FI but keep in mind the complete dependence $F(\beta ; \vartheta, \tau, \gamma)$ on both the qubit degrees of freedom and the parameters $\gamma$ and $\tau$, which drive the coupling. Notice that $F(\beta)$ does not depend on the qubit phase $\varphi$ : its building blocks are, in fact, the probabilities $P(e \mid \beta)$ and $P(e \mid \beta)$, i.e., the diagonal matrix elements, whereas $\varphi$ only appears in off-diagonal matrix elements. Varying the parameter $\vartheta$ from $\pi$ to 0 , we span the entire class of qubit preparation, starting from $|1\rangle$, going through a superposition, and ending in $|0\rangle$.

Let us now consider the system at a fixed value of the temperature, e.g., where the resonator is supposed to be 

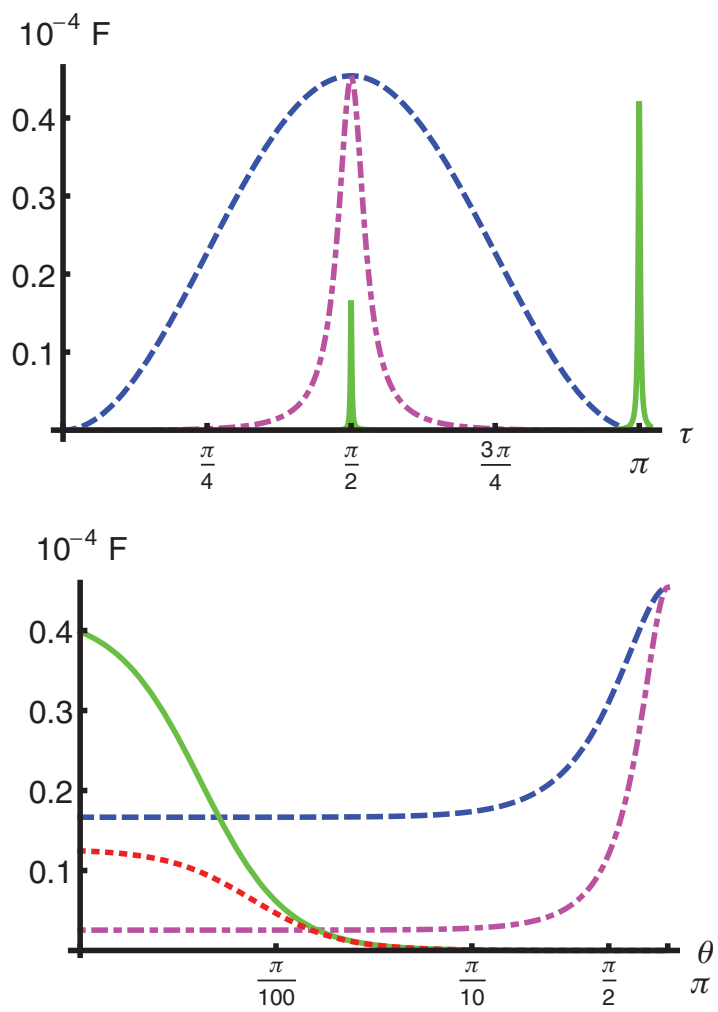

FIG. 1. (Color online) (top) FI for $\beta=10$ as a function of the effective time $\tau$ for different $\vartheta$ values: $\vartheta=\pi$ (dashed blue line), $\vartheta=0.95 \pi$ (dot-dashed magenta line) and $\vartheta=0$ (solid green line). FI takes a pronounced global maximum at $(\vartheta, \tau)=\left(\pi, \frac{\pi}{2}\right)$, while it is possible to see a secondary extremely peaked maximum, which occurs for $\tau=\pi$ and by initially preparing the qubit in $|0\rangle$. (bottom) Log-linear plot of the FI for $\beta=10$ as a function of $\vartheta$ for $\tau=$ $\frac{\pi}{2}$ (dashed blue line), $\tau=\frac{\pi}{2}+\varepsilon$ (dot-dashed magenta line), $\tau=\pi$ (solid green gand), $\tau=\pi+\varepsilon$ (dotted red line), with $\varepsilon=0.01$.

very close to the ground state, say $\beta=10$. The probabilities $p(j \mid \beta)=\varrho_{j j}$ evolve periodically in time according to Eq. (9), as the coupled system undergoes Rabi oscillations. The corresponding behavior of the FI is shown in the top panel of Fig. 1. The FI displays a robust maximum at the optimal time $\tau_{\max }=\frac{\pi}{2}$ for $\vartheta=\pi$, corresponding to initially preparing the qubit in its ground state. This maximum is, at the same time, the global and the smoothest one. In fact, as soon as $\vartheta$ is moved from $\pi$, the FI suddenly drops to zero, except for a sharp peak centered at $\tau_{\max }$, monotonically decreasing with respect to $\vartheta$, as shown in the bottom panel of Fig. 1. Another maximum of the same order of the global one can be found at $(\vartheta, \tau)=(0, \pi)$, but it is extremely peaked, thus representing a bad (unstable) choice for a possible measurement. Upon inspecting the temporal evolution of the excited state probability we found that $p(e \mid \beta)$ has a minimum at $\tau=\tau_{\max }$, a fact that gives us a physical insight on the FI behavior: since our goal is the estimation of a vanishing quantity that carries information about thermal disorder, we expect to find the maximum sensitivity in our predictions where the excitation is most likely stored, as a phonon, in the resonator, i.e., when $p(e \mid \beta)$ is minimum.

Let us now turn our attention to the dependence of the FI on the temperature itself. In Fig. 2 we show, on a logarithmic

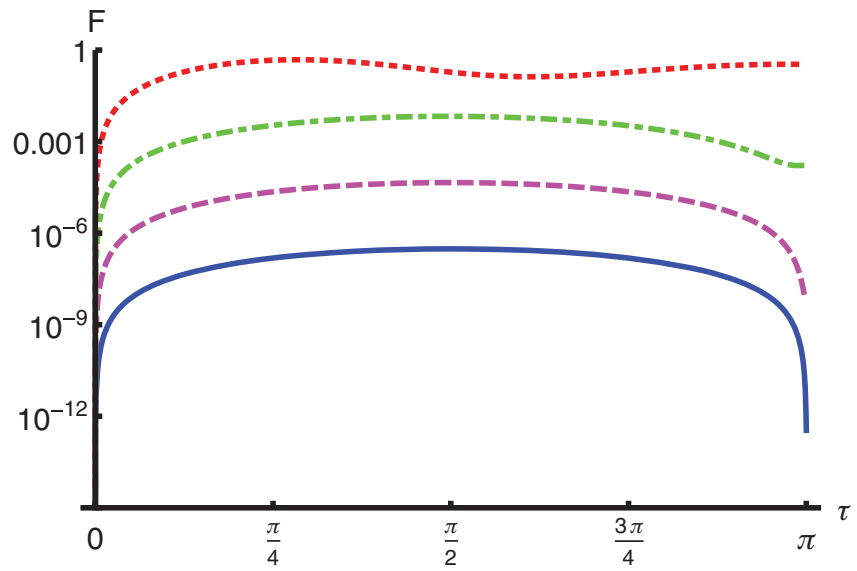

FIG. 2. (Color online) Log-linear plot of the FI as a function of effective time $\tau$ for different values of $\beta$. The qubit is prepared in the ground state $|1\rangle(\vartheta=\pi)$. From bottom to top, $\beta=15$ (solid blue line), $\beta=10$ (dashed magenta line), $\beta=5$ (dot-dashed green line), and $\beta=1$ (dotted red line). Upon raising the temperature the FI no longer keeps a scale-free shape: thermal excitations modify its profile, making it irregular. In particular, the global maximum comes earlier in time.

scale, the temporal evolution of the FI for different values of $\beta$. FI varies over several orders of magnitude, matching our intuition that the closer we are to the ground state, the harder it is to achieve a given precision in the estimation of temperature. Furthermore, upon lowering the temperature, the temporal evolution of $p(j \mid \beta)$ becomes less involved, finally approaching the exactly periodic one of Rabi oscillations, which, in turn, freezes the profile of the FI in a shape independent of the temperature itself.

The qubit preparation $\theta=\pi$ is universally optimal, i.e., it leads to a maximum of the FI independently of the interaction time. After fixing $\theta=\pi$ we have numerically maximized $F(\beta)$ with respect to $\tau$. The solid blue line in the top panel of Fig. 3 is the the log plot of

$$
F_{M}(\beta)=\max _{\tau} F(\beta)
$$

as a function of $\beta$, from which the exponential decrease of the maximum value achieved by the FI for increasing $\beta$ is apparent. The Cramér-Rao inequality immediately relates this fact to an exponential loss of sensitivity moving toward the quantum ground state of the resonator. Another interesting feature that emerges from the maximization is a shift in the value of the optimal interaction time. In the bottom panel of Fig. 3 we can recognize the existence of a steady value for the optimal time $\tau_{\max }=\frac{\pi}{2}$ when approaching the ground state, while for smaller values of $\beta$ the optimal time comes earlier. In fact, the temporal evolution of FI (see Fig. 2) not only predicts an exponential increase of the global maximum when temperatures are raised but also predicts a shift of its location.

\section{B. Effects of detuning}

In this section we take into account the possible existence of a nonzero detuning $\gamma$ between the oscillator and the qubit frequencies. This has two main consequences, which are both illustrated in Fig. 3. On the one hand, the maximum 

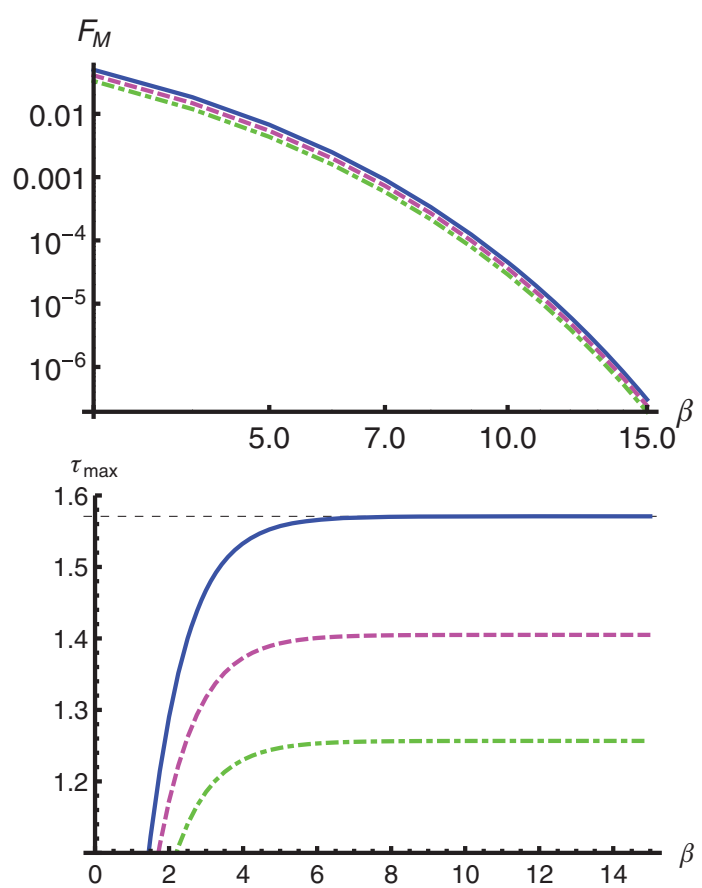

FIG. 3. (Color online) (top) Log-log plot of the FI maximized over $\tau$ as a function of $\beta$, with $\theta=\pi$ for different values of detuning: $\gamma=0$ (solid blue line), $\gamma=1$ (dashed magenta line), and $\gamma=1.5$ (dot-dashed green line). (bottom) The times $\tau_{\max }$ that maximize the FI as a function of $\beta$, with $\theta=\pi$ for different values of $\gamma$ (the same values and color scheme as in the top panel).

achievable value of the FI slightly decreases and, on the other hand, the optimal interaction time $\tau_{\max }$ at which the maximum takes place decreases. Therefore, the best working conditions to achieve the optimal sensitivity in the estimation of $\beta$ correspond to having the qubit and the resonator in resonance. It is also worth noting that $\gamma$ does not represent a critical parameter, as for the initial preparation of the qubit, since the FI dependence on $\gamma$ is smooth. This is shown in the top panel of Fig. 3, where we see that curves corresponding to quite different values of the detuning are almost superposed.

\section{Quantum Fisher information}

In order to assess the performance of the population measurement in the estimation of temperature we have evaluated the QFI of the family $\varrho_{P}(\beta)$. The diagonalization of the probe state has to be carried out numerically; hence, in general, analytical expressions of the QFI are not available. A first fact is that $G(\beta)$ turns out to be independent of the qubit phase $\varphi$, which then does not represent an extra degree of freedom. (erase "whereby more restrictive bounds to the precision on $\operatorname{Var}(\beta)$ are added". Even the optimal qubit preparation for the best conceivable measurement involves control of the parameter $\vartheta$ only.

As we have done for the FI, we start to inspect the QFI behavior for a fixed value of temperature $\beta$ in the resonant case. Also, for the QFI the maximum is achieved by preparing the qubit in the ground state $|g\rangle$ and probing it at the time $\tau_{\max }$ maximizing the FI of the population measurement. In this case the behavior of $G(\beta)$ is identical to that of $F(\beta)$,
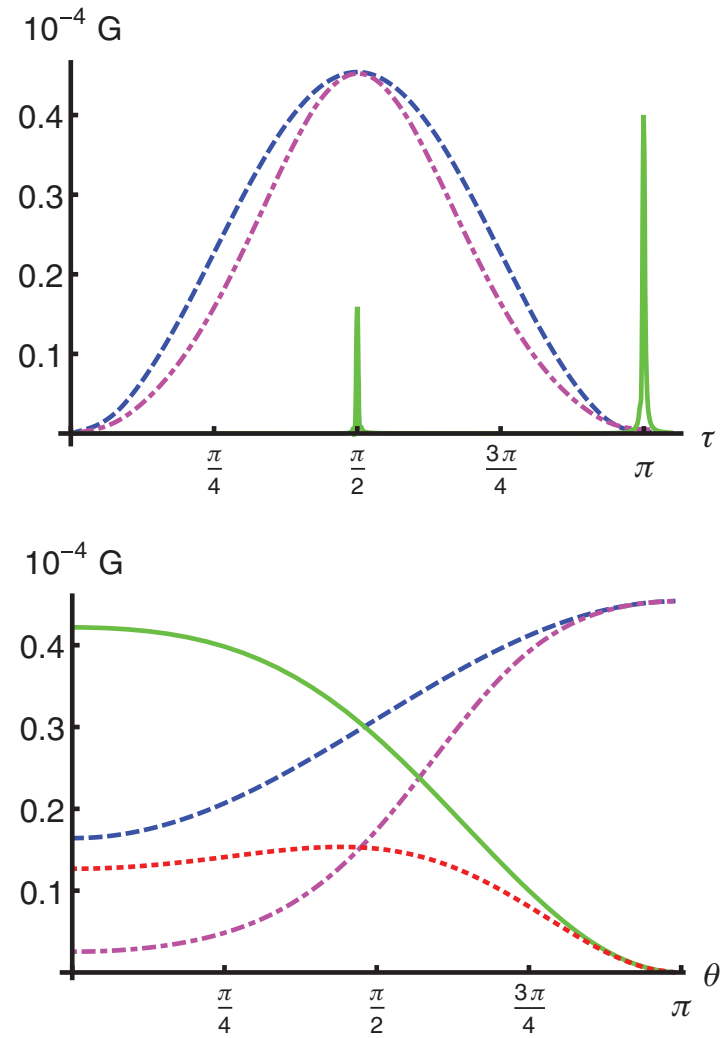

FIG. 4. (Color online) (top) QFI for $\beta=10$ as a function of $\tau$ for $\vartheta=\pi$ (dashed blue line), $\vartheta=0.95 \pi$ (dot-dashed magenta line), and $\vartheta=0$ (solid green line). QFI behaves like FI for $\vartheta=\pi$, leading to the same maximum, while for smaller angles it shows a smoother profile. For angles $0<\vartheta<\pi$ one may find measurements that improve the precision of temperature estimation. (bottom) QFI for $\beta=10$ as a function of $\vartheta$ for $\tau=\frac{\pi}{2}$ (dashed blue line), $\tau=\frac{\pi}{2}+\varepsilon$ (dot-dashed magenta line), $\tau=\pi$ (solid green line), and $\tau=\pi+\varepsilon$ (dotted red line), with $\varepsilon=0.01$.

as is apparent by comparing Figs. 1 and 4 . In other words, for a given value of the parameter $\beta$ in the range explored, the choice $(\vartheta, \tau)=\left(\pi, \tau_{\max }\right)$ makes population measurement optimal. Moreover, the QFI itself reaches its global maximum for that choice. Thus, provided that an optimal estimator is employed, e.g., maximum likelihood in the asymptotic regime, this strategy provides optimality in the sense that inequality (14) is saturated and the right-hand side of QCR is as low as possible.

This conclusion is confirmed upon a closer inspection of the probe state. When $\vartheta=\pi$, the off-diagonal terms vanish, and $\varrho_{P}$ is diagonal, with eigenvalues

$$
\begin{gathered}
\varrho_{+}=\sum_{n=0}^{\infty} p_{n}(\Omega, \beta) \sin ^{2}\left[\sqrt{\gamma^{2}+4 n} \frac{\tau}{2}\right] \frac{n}{n+\gamma^{2} / 4}, \\
\varrho_{-}=1-\varrho_{+} .
\end{gathered}
$$

As a consequence, the $\mathrm{QFI}$ reduces to

$$
G(\beta ; \pi, \tau, \gamma)=\frac{\left(\partial_{\beta} \varrho_{+}\right)^{2}}{\varrho_{+}}+\frac{\left(\partial_{\beta} \varrho_{-}\right)^{2}}{\varrho_{-}},
$$

which coincides with the FI ruling the estimation of $\beta$ via population measurement. 

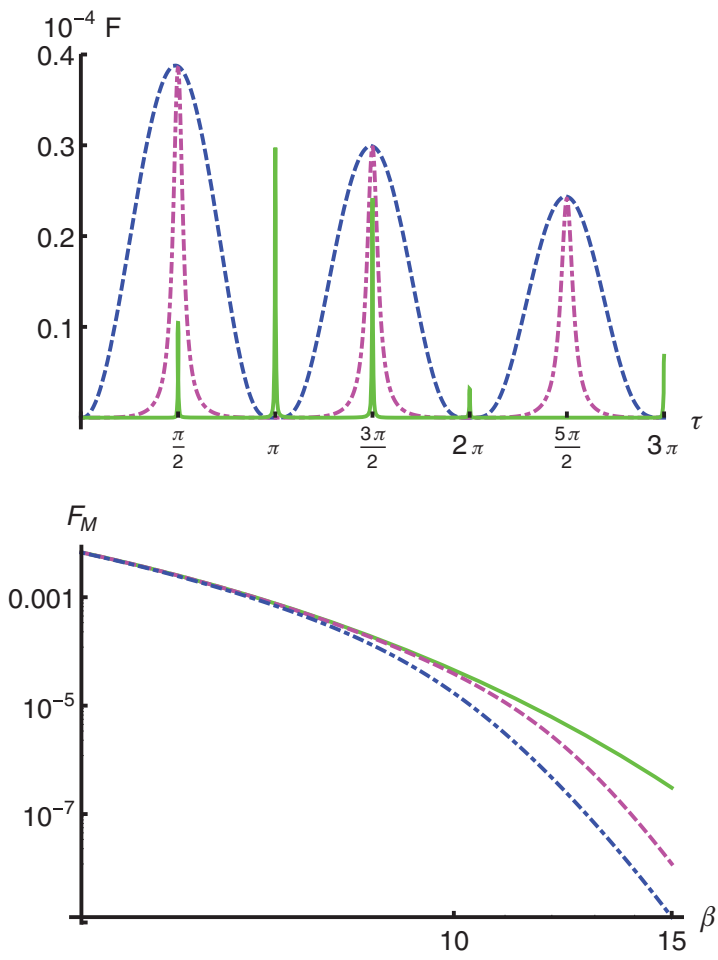

FIG. 5. (Color online) (top) Fisher information $F(\beta)$ for $\beta=10$ as a function of $\tau$ in the presence of decoherence and for different qubit preparations. The decoherence parameters are chosen as $a=$ 0.1 and $b=10^{-5}$. Dashed blue line stands for $\vartheta=\pi$, dot-dashed magenta line stands for $\vartheta=0.95 \pi$, and solid green line stands for $\vartheta=0$. Having included the decoherence treatment enables us not to restrict the evolution to the first Rabi half period. (bottom) Log-log plot of the Fisher information $F_{M}(\beta)$ maximized over the interaction time and in the presence of decoherence as a function of $\beta$ and for fixed $\vartheta=\pi$ for $b=0$ (solid green line), $b=10^{-5}$ (dashed magenta line), and $b=10^{-4}$ (dot-dashed blue line).

Nevertheless, some striking differences emerge between the performances of population measurement and that of the optimal one if the qubit is not prepared in the optimal (ground) state. In the bottom panel of Fig. 4 we show $G(\beta)$ as a function of $\tau$ for different values of $\vartheta$ : for $\vartheta<\pi$ the decrease of $G$ is definitely smoother than that of $F$, and thus, in principle, some measurement may be found, making the initial preparation a less critical parameter. Moreover inspecting the cut of the QFI along $\tau=\pi$, we note that the maximum in $\vartheta=0$ becomes more achievable compared to the one of $F(\beta)$. All these features suggest that for qubit preparations different from the ground state there will be a significant difference between the precision provided by population measurement and the optimal one implementable in the system. On the other hand, being the overall maximum achievable with population measurement, our results indicate that the achievement of the ultimate bound to precision allowed by quantum mechanics is in the capabilities of current technology.

\section{Effects of decoherence}

In this section we discuss the solution of the reduced qubit dynamics in the presence of dissipative decoherence [see Eq. (10)] and inspect the corresponding behavior of the FI.
For the sake of simplicity we consider zero detuning. Analog results are obtained when including the detuning.

The probabilities $p(j \mid \beta)=\tilde{\varrho}_{j j}$ are damped so that, waiting for a sufficiently long time, whose value depends on $a$ and $b$, we would find them to be identically $1 / 2$, or equally stated, the dynamical evolution brings the state to the maximally mixed one. The contribution of decoherence is of the kind $\exp \left[-b(1+n)^{a} \tau\right]$ for every $n$, where $b$ has been rescaled in coupling units $b \longmapsto b / \lambda$. Being a multiplicative coefficient, as soon as $b$ is different from zero, the exponential term will participate in killing the sums. Our calculations show a relevant dependence of the FI on the parameter $b$, namely, values $b \approx 10^{-5}$ are enough to produce visible effects, while varying $a$ in the range $(0,1)$ does not deeply influence FI behavior.

In Fig. 5 we show the temporal evolution of the FI for $\beta=10$ in the presence of decoherence and for different initial preparations of the qubit. In the Hamiltonian regime for large $\beta$ the resonator is close to the ground state, the evolution of $p(j \mid \beta)$ is periodic, and hence, due to Eq. (12), the same is true for the FI. Upon incorporating decoherence we see that FI decays at a rate depending on $b$, and thus an irreversible dynamics emerges, which matches the physical evidence of a limited coherence time. On the other hand, a clear maximum at $\tau=\pi / 2$ still appears, with a slightly decreased value of $F(\beta)$. In the bottom panel of Fig. 5 we show the maximum value $F_{M}=\max _{\tau} F(\beta)$ for different values of the decoherence parameter. As is apparent from the (log-log) plot for high temperature (smaller $\beta$ ) the effect of decoherence is negligible, whereas for increasing $\beta$ the effect becomes more and more relevant.

\section{CONCLUSIONS}

The temperature of a physical object cannot be directly measurable. On the other hand, it can be regarded as a parameter whose value can be indirectly inferred by measuring some proper observable and then suitably processing the outcomes, an inference procedure usually referred to as an estimation procedure. In the case of a micromechanical oscillator with an isolated vibrational mode, effective schemes have been suggested and realized [11] that rely on coupling the resonator to a superconducting qubit and probing the latter using population measurements. In other words, the qubit is employed as a quantum thermometer to demonstrate that the resonator has been cooled to its quantum ground state. In this paper we have analyzed in detail qubit thermometry in these systems, i.e., the estimation of temperature via quantum-limited measurements performed on the qubit. In the framework of quantum estimation theory we have analyzed precision as a function of both the qubit initial preparation and the interaction parameters, and we have evaluated the limits to precision posed by quantum mechanics to qubit thermometry.

We have computed the FI for population measurement, which is the appropriate figure of merit to assess the precision of estimation, and have found that its maximum, and hence the minimum variance in the estimated temperature, is achieved by preparing the qubit in the ground state and probing it at an emergent time $\tau_{\max }$, which is predictable. Furthermore, we have analyzed in detail how the maximum depends on the temperature itself, on the detuning, and on the noise parameter 
when one takes into account nondissipative decoherence. In order to evaluate the ultimate bound allowed by quantum mechanics to the sensitivity of temperature estimation, we have also computed the quantum Fisher information. We found that QFI is maximized for the same choice of qubit preparation and measurement time of the FI and that for these common values the maxima of FI and QFI coincide. We thus conclude that population measurement is optimal for temperature estimation.

The range of parameters addressed in our analysis is that of recent experimental implementations [11]. We thus conclude that optimal estimation of temperature can be done with current technology. Since the FI of population measurement and the QFI of the model both decrease with the decrease of temperature, the estimation of lower temperatures will be intrinsically less precise. On the other hand, since there are regimes, also in the presence of decoherence, where the maxima of the FI and the QFI are reasonably smooth as a function of the qubit preparation and of the interaction time we do not expect any "no-go" theorem for temperature estimation. In other words, we expect that optimal estimation of lower resonator temperatures, perhaps achievable with further experimental advances, will be still possible with population measurements. On the other hand, "optimality" will correspond to an inherently less precise procedure compared to the case of higher temperature.

Our analysis shows the optimality of feasible qubit thermometry in providing quantum benchmarks for high-precision temperature measurement as well as an efficient operational quantification of temperature for mechanical modes lying arbitrarily close to their ground state. In other words, achievement of the ultimate bound to precision allowed by quantum mechanics is within the capabilities of current technology. Our results also confirm that QET is a useful tool for assessing and comparing inference procedures arising in quantumlimited measurements [42], even when mesoscopic objects are involved.

\section{ACKNOWLEDGMENT}

This work has been partially supported the CNR-CNISM agreement.
[1] J. M. Courty, A. Heidmann, and M. Pinard, Eur. Phys. J. D 17, 399 (2001)

[2] A. D. Armour, M. P. Blencowe, and K. C. Schwab, Phys. Rev. Lett. 88, 148301 (2002).

[3] A. N. Cleland and M. R. Geller, Phys. Rev. Lett. 93, 070501 (2004).

[4] M. D. LaHaye, P. Buu, B. Camarota, and K. C. Schwab, Science 304, 74 (2004).

[5] M. Blencowe, Phys. Rep. 395, 159 (2004).

[6] I. Martin, A. Shnirman, L. Tian, and P. Zoller, Phys. Rev. B 69, 125339 (2004).

[7] D. Kleckner and D. Bouwmeester, Nature (London) 444, 75 (2006).

[8] A. Schliesser, R. Riviere, G. Anetsberger, O. Arcizet, and T. J. Kippenberg, Nat. Phys. 4, 415 (2008).

[9] C. A. Regal, J. D. Teufel, and K. W. Lehnert, Nat. Phys. 4, 555 (2008).

[10] T. Rocheleau, T. Ndukum, C. Macklin, J. B. Hertzberg, A. A. Clerk, and K. C. Schwab, Nature (London) 463, 7275 (2010).

[11] A. D. O’Connell et al., Nature (London) 464, 697 (2010).

[12] A. Monras, Phys. Rev. A 73, 033821 (2006).

[13] S. Olivares and M. G. A. Paris, J. Phys. B 42, 055506 (2009).

[14] M. Aspachs, J. Calsamiglia, R. Munoz-Tapia, and E. Bagan, Phys. Rev. A 79, 033834 (2009).

[15] M. G. Genoni, S. Olivares, and M. G. A. Paris, Phys. Rev. Lett. 106, 153603 (2011).

[16] M. G. Genoni, P. Giorda, and M. G. A. Paris, Phys. Rev. A 78, 032303 (2008).

[17] G. Brida, I. P. Degiovanni, A. Florio, M. Genovese, P. Giorda, A. Meda, M. G. A. Paris, and A. Shurupov, Phys. Rev. Lett. 104, 100501 (2010).

[18] G. Brida, I. P. Degiovanni, A. Florio, M. Genovese, P. Giorda, A. Meda, M. G. A. Paris, and A. P. Shurupov, Phys. Rev. A 83, 052301 (2011).

[19] M. Sarovar and G. Milburn, J. Phys. A 39, 8487 (2006).
[20] M. Hotta, T. Karasawa, and M. Ozawa, Phys. Rev. A 72, 052334 (2005).

[21] A. Monras and M. G. A. Paris, Phys. Rev. Lett. 98, 160401 (2007).

[22] A. Fujiwara, Phys. Rev. A 65, 012316 (2001).

[23] Z. Ji, G. Wang, R. Duan, Y. Feng, and M. Ying, IEEE Trans. Inf. Theory 54, 5172 (2008).

[24] S. Boixo and A. Monras, Phys. Rev. Lett. 100, 100503 (2008).

[25] P. Zanardi, M. G. A. Paris, and L. Campos Venuti, Phys. Rev. A 78, 042105 (2008); C. Invernizzi, M. Korbman, L. Campos Venuti, and M. G. A. Paris, ibid. 78, 042106 (2008).

[26] S. Campbell, M. Paternostro, S. Bose, and M. S. Kim, Phys. Rev. A 81, 050301(R) (2010).

[27] M. Paternostro, S. Gigan, M. S. Kim, F. Blaser, H. R. Bohm, and M. Aspelmeyer, New J. Phys. 8, 107 (2006).

[28] A. Monras and F. Illuminati, Phys. Rev. A 81, 062326 (2010).

[29] A. Monras and F. Illuminati, Phys. Rev. A 83, 012315 (2011).

[30] B. B. Mandelbrot, Ann. Math. Stat. 33, 1021 (1962); J. Math. Phys. 5, 164 (1964); Phys. Today 71 (1989).

[31] C. W. Helstrom, Quantum Detection and Estimation Theory (Academic, New York, 1976); A. S. Holevo, Statistical Structure of Quantum Theory, Lecture Notes in Physics Vol. 61, (Springer, Berlin, 2001).

[32] S. L. Braunstein and C. M. Caves, Phys. Rev. Lett. 72, 3439 (1994); S. L. Braunstein, C. M. Caves, and G. J. Milburn, Ann. Phys. (NY) 247, 135 (1996).

[33] A. Fujiwara, Department of Mathematical Engineering and Information Physics, University of Tokyo, METR Report No. 94-08, 1994 (unpublished).

[34] D. C. Brody and L. P. Hughston, Proc. R. Soc. London, Ser. A 454, 2445 (1998); 455, 1683 (1999).

[35] S. Amari and H. Nagaoka, Methods of Information Geometry, Translations of Mathematical Monographs Vol. 191 (American Mathematical Society, Providence, 2000). 
[36] M. G. A. Paris, Int. J. Quant. Inf. 7, 125 (2009).

[37] J. Gemmer, M. Michel, and G. Mahler, Quantum Thermodynamics, Lecture Notes in Physics Vol. 784 (Springer, Berlin, 2009).

[38] C. H. Webster, National Physical Laboratory, NPL Report No. DEM-TQD-007, 2006 (unpublished).
[39] T. Jahnke, S. Lanery, and G. Mahler, Phys. Rev. E 83, 011109 (2011).

[40] T. L. Schmidt, K. Borkje, C. Bruder, and B. Trauzettel, Phys. Rev. Lett. 104, 177205 (2010).

[41] D. M. Meekhof, C. Monroe, B. E. King, W. M. Itano, and D. J. Wineland, Phys. Rev. Lett 76, 1796 (1996).

[42] T. M. Stace, Phys. Rev. A 82, 011611 (2010). 